\title{
Evidence for UHECR origin in starburst galaxies
}

\section{Luis A. Anchordoqui}

Department of Physics \& Astronomy, Lehman College, CUNY, NY 10468, USA

Department of Physics, Graduate Center, City University of New York, NY 10016, USA

Department of Astrophysics, American Museum of Natural History, NY 10024, USA

E-mail: luis.anchordoqui@gmail.com

\section{Jorge F. Soriano*}

Department of Physics \& Astronomy, Lehman College, CUNY, NY 10468, USA

Department of Physics, Graduate Center, City University of New York, NY 10016, USA

E-mail: jfdezsoriano@gmail.com

The quest for the origin(s) of ultra-high-energy cosmic rays (UHECRs) continues to be a far-reaching pillar of high energy astrophysics. The source scrutiny is mostly based on three observables: the energy spectrum, the nuclear composition, and the distribution of arrival directions. We show that each of these three observables can be well reproduced with UHECRs originating in starburst galaxies.

36th International Cosmic Ray Conference - ICRC2019 -

July 24 - August 1, 2019

Madison, Wisconsin, USA

${ }^{*}$ Speaker. 
Starburst galaxies are observed to be forming stars at an unusually fast rate (about $10^{3}$ times greater than in a normal galaxy). The areas of high activity can be spread throughout the galaxy, but most star forming regions are observed in a small sector around the nucleus. The starburst activity usually drives galactic-scale outflows or "superwinds" that may be responsible for removing metals from the galactic disk and polluting the intergalactic medium with ultra-high-energy $\left(E \gtrsim 10^{9} \mathrm{GeV}\right)$ cosmic ray (UHECR) nuclei [1,2]. Starburst superwinds are powered by massive star winds and by core collapse supernovae which collectively create hot $\left(T \lesssim 10^{8} \mathrm{~K}\right)$ bubbles of metal-enriched plasma within the star forming regions. The over-pressured bubbles expand, sweep up cooler ambient gas, and eventually blow out of the disk into the halo, providing a profitable arena for the formation of collisionless plasma shock waves, in which UHECRs can be accelerated by bouncing back and forth across the shock. Herein we present additional support for this idea by confronting the predictions of the model with experimental data.

Specific assumptions are made, in that we consider diffusive shock acceleration on a distribution of particles at multiple parallel shocks (in which both the magnetic field and the upstream and downstream plasma flows are always perpendicular to the plane of the shock front) [3]. Note since the magnetic field has components only along the direction in which the shock propagates the Rankine-Hugoniot jump conditions are hydrodynamic in character [2]. At each shock a new distribution of particles is injected and accelerated, and the particles injected at earlier shocks are re-accelerated further. Adiabatic decompression occurs after each shock. We show that these considerations reduce the time constraint on the acceleration region, while addressing the criticism on the model raised in [4]. Moreover, the presence in the wind of many shocks changes the particle spectrum from that produced by a single shock [3]. Summing over an infinite number of identical shocks, with fresh injection at each shock and decompression between the shocks, does produce a power-law momentum distribution $f_{\infty}(p) \propto p^{-3}$, which is flatter than that produce by a single shock $f(p) \propto p^{-4}$, and better reproduce observations.

The UHECR spectrum can be roughly described by a twice-broken power law. The first break is a hardening of the spectrum, known as "the ankle." The second is an abrupt softening of the spectrum, which (i) may be interpreted as the long-sought GZK cutoff [5], or (ii) may correspond to the "end-of-steam" for cosmic accelerators [6]. Herein we introduce a complementary explanation (iii) in which GZK interactions at the source constrain the maximum energy of the nuclei. Note that (iii) is markedly different from (ii) because for a nucleus of charge $Z e$ and baryon number $A$, the maximum energy of acceleration capability of the sources grows linearly in $Z$, while the energy loss per distance traveled decreases with increasing $A$. The ankle energy and the corresponding change in the power-law spectral index are measured with high precision. The existence of the flux suppression is also firmly established. The differential energy spectra measured by the Telescope Array (TA) experiment and the Pierre Auger Observatory (Auger) agree within systematic errors below $E \sim 10^{10} \mathrm{GeV}$; at higher energies, TA observes more cosmic rays than would be expected if the spectral shape were the same as that seen by Auger [2]. The flux suppression observed in Auger data is at $E \sim 10^{10.6} \mathrm{GeV}$, whereas the one observed in TA data is at $E \sim 10^{10.73} \mathrm{GeV}$. 
The TA Collaboration has interpreted their data as implying a light primary composition (mainly $p$ and $\mathrm{He}$ ) from $10^{9.1}$ to $10^{10.6} \mathrm{GeV}$. The Auger Collaboration, using post-LHC hadronic interaction models, reports a composition becoming light up to $10^{9.3} \mathrm{GeV}$ but then becoming heavier above that energy, with the mean mass intermediate between protons and iron at $10^{10.5} \mathrm{GeV}$. Auger and TA have also conducted a thorough joint analysis and state that, at the current level of statistics and understanding of systematics, both data sets are compatible with being drawn from the same parent distribution, and that the TA data is compatible both with a protonic composition below $10^{10} \mathrm{GeV}$ and with the mixed composition above $10^{10} \mathrm{GeV}$ as reported by Auger. However, Auger data are more constraining and not compatible with the pure protonic option available with TA alone. For further details on the mass spectrum, see e.g. [2].

The high frequency spectral fall-off and the shape of the spectrum at and below the corner frequency are critical to asses the characteristics of the source spectra. In particular, a simultaneous fit to the spectrum and the elongation rate requires hard source spectra $\propto E^{-\gamma}$, with $1.0 \lesssim \gamma \lesssim 1.5$ [2]. The differential energy spectrum $d N / d E \propto E^{-\gamma}$ is related to the phase space distribution in momentum space by $d N=4 \pi p^{2} f_{\infty}(p) d p$, yielding good agreement with Auger data. The constraint on the source spectral index would be relaxed if the number of UHECR sources increases at low redshifts (for such an unusual redshift evolution softer source spectra with $d N=4 \pi p^{2} f(p) d p$ are favored) [7].

The Auger Collaboration has found an indication of a possible correlation between UHECRs of $E>10^{10.6} \mathrm{GeV}$ and nearby starburst galaxies, with an a posteriori (post-trial) chance probability in an isotropic cosmic ray sky of $4.2 \times 10^{-5}$ ( $4 \sigma$ significance) [8]. The energy threshold of largest statistical significance coincides with the observed suppression in the spectrum, implying that when we properly account for the barriers to UHECR propagation in the form of energy loss mechanisms [5] we obtain a self consistent picture for the observed UHECR horizon. The TA Collaboration has reported that with their current statistics [9] they cannot make a statistically significant corroboration or refutation of the reported possible correlation between UHECRs and starburst galaxies. However, TA has recorded a statistically significant excess in cosmic rays, with energies above $10^{10.75} \mathrm{GeV}$, above the isotropic background-only expectation [10]. This is colloquially referred to as the "TA hot-spot." The excess is centered at Galactic coordinates $(l, b) \simeq\left(177^{\circ}, 50^{\circ}\right)$, spanning a region of the sky with $\sim 20^{\circ}$ radius. The chance probability of this hot spot in an isotropic cosmic ray sky was calculated to be $3.7 \times 10^{-4}$ ( $3.4 \sigma$ significance). The possible association of the TA hot-spot with the nearby (3.4 Mpc away) starburst galaxy M82 has not gone unnoticed [2]. We have seen that starburst galaxies can accommodate two of the main observables in UHECR physics: the nuclear composition and the distribution of arrival directions. We turn now to discuss the acceleration process in starburst superwinds, while exploring also whether this model can accommodate the shape of the source spectra.

With the motivation loaded we can now look at the calculations. The UHECR emission from starbursts is attributed to shock accelerated particles. We describe the acceleration of these particles through the energy gain $g \equiv d E / d t$. We consider acceleration at superwindembedded shocks in which the gain $g_{\mathrm{SW}}$ can be described by $g_{\mathrm{SW}}=\xi E / T_{\text {cycle}}$, where $T_{\text {cycle }}=$ $4 \kappa\left(1 / u_{1}+1 / u_{2}\right)$ is the duration of each acceleration cycle, $\xi \sim \frac{4}{3}\left(u_{1}-u_{2}\right)$ is the fractional 
energy gain per encounter, $\kappa=\frac{1}{3} R_{\mathrm{L}} \sim \frac{1}{3} \frac{E}{\mathrm{ZeB}}$ is the diffusion coefficient, $R_{\mathrm{L}}$ is the Larmor radius, and $u_{1}$ and $u_{2}$ are the upstream and downstream gas velocities [2]. For simplicity, we demand that any two shocks do not propagate simultaneously. Studies of more general set-ups, with shock correlation effects, are underway and will be presented elsewhere. For typical superwind parameters $u_{2}=u_{1} / 4$ and $u \equiv u_{1}=v_{\infty} \sim 1.8 \times 10^{3} \mathrm{~km} / \mathrm{s}$, the energy gain $g_{\mathrm{SW}}^{(\mathrm{Z})}(B)=\frac{3}{20} \mathrm{ZeBu}{ }^{2}$, produces a linear increase of energy as a function of time $\mathcal{E}\left(E_{0}, t_{0}, t\right)=$ $E_{0}+g_{\mathrm{SW}}^{(\mathrm{Z})}\left(t-t_{0}\right)$, for a fixed magnetic field. Thus, for an accelerator of size $R_{\mathrm{SW}} \sim 8 \mathrm{kpc}$, the maximum energy is

$$
E_{\max } \sim g_{\mathrm{SW}}^{(\mathrm{Z})} \Delta t
$$

where $\Delta t=t-t_{0}$. For a single shock, we have $\Delta t=R_{\mathrm{SW}} / u$. Substituting for $\Delta t$ in into the relation for the maximum energy leads to the Hillas maximum rigidity [11]

$$
\mathcal{R}_{\mathrm{H}, \max } \sim 10^{9}(u / c)\left(\frac{B}{\mu \mathrm{G}}\right)\left(\frac{R_{\mathrm{SW}}}{\mathrm{kpc}}\right) \mathrm{GV} .
$$

To develop some sense of the orders of magnitude involved, we assume that M82 and NGC 253 typify the nearby starburst population. The magnetic field $B$ carries with it an energy density $B^{2} /(8 \pi)$, and the flow carries with it an energy flux $>u B^{2} /(8 \pi)$. This sets a lower limit on the rate at which the energy is carried by the out-flowing plasma, $L_{B} \sim u R_{\mathrm{SW}}^{2} B^{2} / 8$, and which must be provided by the source. The flux carried by the outgoing plasma is a model dependent parameter, which can be characterized within an order of magnitude. More concretely, $0.035 \lesssim L_{B} / L_{\text {IR }} \lesssim 0.35$, where $L_{\text {IR }} \sim 10^{43.9} \mathrm{erg} / \mathrm{s}$ is the infrared luminosity. The lower limit of $L_{B}$ corresponds to the estimate in [12] considering a supernova rate of $0.07 \mathrm{yr}^{-1}$, whereas the upper limit concur with the estimate in [13], and could be obtained considering a supernova rate of $0.3 \mathrm{yr}^{-1}$ [14] while pushing other model parameters to the most optimistic values. Then $L_{B}$ yields a magnetic field strength in the range

$$
15 \lesssim B / \mu \mathrm{G} \lesssim 150 .
$$

Substitution of (3) into (2) leads to $10^{8.9} \lesssim \mathcal{R}_{\mathrm{H}, \max } / \mathrm{GV} \lesssim 10^{9.9}$. Taking this at face value, one would tend to interpret that starburst superwinds struggle to accelerate light nuclei $(Z \lesssim 8)$ up to the highest observed energies [4]. Note, however, that in the case of multiple shocks the time scale $\Delta t$ is not constrained by the ratio of the size of the accelerator to the shock velocity, but rather by the lifetime of the source $\Delta t \sim \tau$ [1]. Then, for UHECRs experiencing the effect of multiple shocks, the maximum rigidity is set by the Larmor radius,

$$
\mathcal{R}_{\mathrm{L}, \max } \sim 10^{9}\left(\frac{B}{\mu \mathrm{G}}\right)\left(\frac{R_{\mathrm{SW}}}{\mathrm{kpc}}\right) \mathrm{GV},
$$

with an external constraint set by the energy loss. It is this that we now turn to suty.

The final energy after the acceleration process for a fixed species $(A, Z)$ is given by the competition between the superwind acceleration and the possibility that a given nucleus suffers a photodisintegration and becomes a new species $\left(A^{\prime}, Z^{\prime}\right)$ after loosing one or several nucleons. A nucleus injected into the superwind at a time $t_{0}$ has probability $d P=f\left(t_{0}, t\right) d t$ 
to suffer a photodisintegration in the time in the interval $[t, t+d t]$, where

$$
f\left(t_{0}, t\right)=\frac{\mathcal{F}\left(t_{0}, t\right)}{\tau\left[\mathcal{E}\left(E_{0}, t_{0}, t\right)\right]}, \quad \mathcal{F}\left(t_{0}, t\right)=\exp \left(-\int_{t_{0}}^{t} \frac{d t^{\prime}}{\tau\left(\mathcal{E}\left(E_{0}, t_{0}, t^{\prime}\right)\right)}\right),
$$

and $\tau(E)$ is the mean free path for the nucleus at a given energy. The accelerating nucleus will gain energy until it eventually suffers a photodisintegration at a time $t$ distributed following (5).

The photodisintegration rate depends on the energy density of the ambient radiation field. This is governed by the spatial distribution of photons, including both those from the cosmic microwave background $(\mathrm{CMB})$ and stellar radiation fields. For compact regions near the galaxy core, starbursts exhibit an energy density in their stellar radiation fields which may exceed (or be comparable to) that of the CMB, but at the superwind scale $R_{\mathrm{SW}}$ starlight is expected to have a negligible energy density compared to that of the CMB [15]. For our calculations, the contribution from the stellar radiation is unimportant and therefore neglected.

In order to describe the energies that can be achieved through superwind acceleration, we consider the probability $d P=h\left(E_{0}, E\right) d E$ for the photodisintegration to happen at an energy in $[E, E+d E]$. Comparing with (5), the distribution for the final energy is

$$
h\left(E_{0}, E\right)=\frac{\mathcal{H}\left(E, E_{0}\right)}{g_{\mathrm{SW}}^{(Z)} \tau(E)}, \quad \text { with } \quad \mathcal{H}\left(E_{0}, E\right)=\exp \left(-\int_{E_{0}}^{E} \frac{d E^{\prime}}{g_{\mathrm{sw}}^{(\mathrm{Z})} \tau\left(E^{\prime}\right)}\right) .
$$

The CMB mean free path is described as [2]

$$
\tau(E)=\left[\frac{c}{4 \pi^{2}}\left(\frac{m}{\hbar c E}\right)^{3} \int_{0}^{\infty} \frac{J(\varepsilon)}{e^{\varepsilon / k T^{\prime}(E)}-1} d \varepsilon\right]^{-1},
$$

where $T^{\prime}(E)=2 E T / A m c^{2}, m$ is the proton mass, $T$ is the CMB temperature,

$$
J(\varepsilon)=\int_{0}^{\varepsilon} \varepsilon^{\prime} \sigma\left(\varepsilon^{\prime}\right) d \varepsilon^{\prime}
$$

and where $\sigma\left(\varepsilon^{\prime}\right)$ is the cross-section for photo-disintegration by a photon of energy $\varepsilon^{\prime}$ in the rest frame of the nucleus. The $\mathcal{H}$ functions have a decreasing sigmoid shape, as can be seen in Fig. 1. This allows to define a cutoff energy $E_{c}$ at the point of their largest decrease rate, which corresponds to the peak of the $h$ functions, shown in Fig. 1 as well. This condition reads

$$
E_{c}=\underset{E>E_{0}}{\arg \max }\left(-\frac{d \mathcal{H}\left(E_{0}, E\right)}{d E}\right)=\underset{E>E_{0}}{\arg \max } h\left(E_{0}, E\right) .
$$

It can be rewritten in terms of the mean free path as

$$
1+\left.g_{\mathrm{sw}}^{(\mathrm{Z})} \frac{d \tau(E)}{d E}\right|_{E=E_{c}}=0,
$$

where the independence of $E_{c}$ on $E_{0}$ has been made explicit. The values of the cutoff energy are shown in Fig. 2 for the region of interesting $B$-strengths. The dispersion 

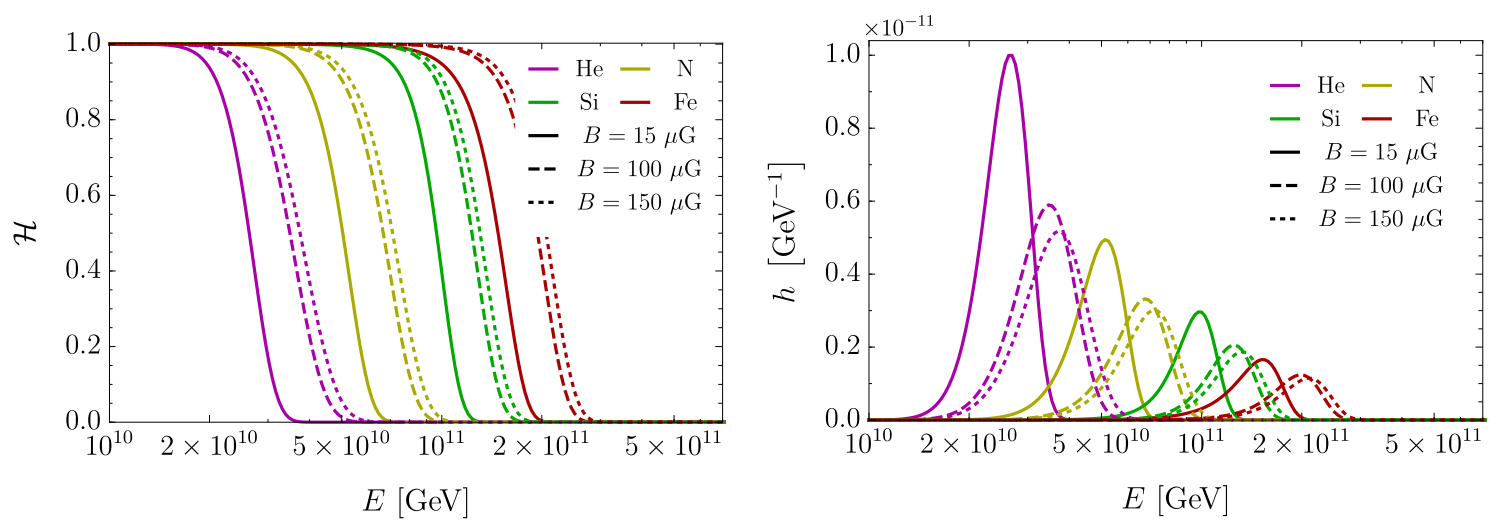

Figure 1: Survival probability (left) and probability density for the energy at which the photodisintegration happens (right), for the four considered nuclei.
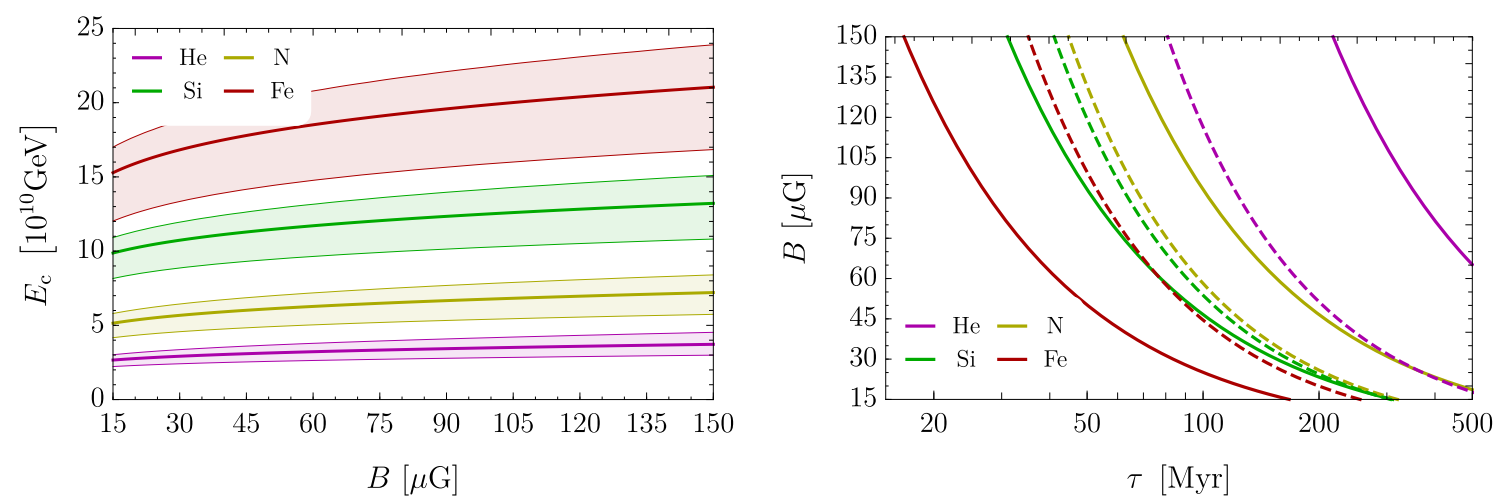

Figure 2: Cutoff energy as a function of the magnetic field for the four considered nuclei (left), and parameter space for the source for a maximum energy of $10^{11} \mathrm{GeV}$, and limits (dashed) imposed by $\mathrm{CMB}$ photodisintegration (right).

around the peak of $h$ suggests that particles of energies above $E_{c}$ might be achieved at the source. In Fig. 2 we show bands containing the $68 \%$ of the probability (i.e. such that $\mathcal{H} \sim 0.16$ and $\mathcal{H} \sim 0.84$ ), which means that nuclei have around $16 \%$ probability of reaching energies above (below) the top (bottom) band. Further calculations show that nitrogen nuclei have a probability of around $7 \%$ of reaching energies above $10^{10.95} \mathrm{eV}$ and $1 \%$ above $10^{11} \mathrm{GeV}$ for $B \sim 150 \mu \mathrm{G}$. The sharp suppression of the probability function with rising energy can accommodate a steeply falling spectrum if sillicon-type nuclei are much less abundant than CNO-type nuclei. A detailed study of this function with predictions on nuclear composition to accommodate the observed spectrum on Earth will be presented elsewhere.

The relevance of $\mathrm{CMB}$ photodisintegration at a certain source depends on the interplay between the lifetime of the source and the mean free path. For short living sources, the energies achieved will not be high enough for the CMB to play a role, and the maximum energy for a certain species will be determined solely due to the lifetime $\tau$ and the magnetic field $B$ as in (1). This relation, equivalently written as $B \propto E_{\max } / \tau$, allows to study the 
parameter space $(\tau, B)$ that would allow to reach a certain energy $E_{\max }$. Nevertheless, as the lifetime increases, the available energies will be limited by photodisintegration processes and, given a maximum energy, it will not always be possible to find a pair $(\tau, B)$ able to provide such energy, since the CMB interactions would produce a cutoff before that energy is reached. In Fig. 2 we explore this parameter space for a maximum energy of $10^{11} \mathrm{GeV}$. The continuous lines follow (1), while the dashed lines define the regions (on the right) that are not accessible due to the previous criterion. It can be seen that on average no pair $(\tau, B)$ would be able to accelerate helium or nitrogen nuclei above that energy, while the opposite is true for silicon and iron nuclei.

In summary, we have re-examined the acceleration of UHECRs in starburst superwinds endowed with multiple, non-simultaneous, propagating shocks. Particles gain energy when they pass through the shock back and forth after being scattered by the flowing plasma. To calculate the maximum energy we must consider not only particles which are accelerated by a single shock but also particles which undergo many shock encounters, each of which further accelerates the particles. There are two length scales which are important for particle acceleration by multiple shocks: the mean free path for high energy particles and the distance between shocks in the superwind. In our approximation, only the first scale is relevant. We have shown that the particle's maximum energy is set by a balance equation driven by the source lifetime and UHECR interactions with the CMB. This gives specific characteristics for the source emission spectra, providing a new interpretation of the observed suppression in the UHECR spectrum.

Up until now, there were two competing classes of models to explain the observed suppression in the energy spectrum. The competing models are: (i) the GZK cutoff due to the UHECR interaction with the CMB during propagation [5], and (ii) the disappointing model [6] wherein it is postulated that the end-of-steam for cosmic accelerators $\propto E_{\max } / Z$ is coincidentally near the putative GZK cutoff. More concretely, conventional UHECR source models presuppose that particle acceleration takes place at sites distributed similarly to the matter distribution in the universe, with energy loss processes during propagation leading to the observed flux suppression (GZK cutoff). However, the most recent data seem to indicate that the uppermost end of the cosmic ray energy spectrum is dominated by nucleus-emitting- sources, possibly within the GZK horizon, for which the upper limit of particle acceleration almost coincides with the energy of the GZK suppression. In contrast to conventional expectations, models in category (ii) suggest that the emission of these sources would be characterized by a harder power-law spectrum with the different mass components exhibiting a rigidity-dependent maximum injection energy $E_{\max } / Z$ of a few EeV. Herein, we have introduced an alternative possibility (iii) in which the maximun energy is driven by GZK interactions, but as in (ii) the observed suppression of the energy spectrum mainly stems from the source characteristics rather than being the imprint of particle propagation through the CMB. Note that (iii) is markedly different from (ii) because the maximum energy of acceleration capability of the sources grows linearly in $Z$, while the energy loss per distance traveled decreases with increasing $A$.

Class (iii) models have very particular predictions, which can be easily distinguished from those in models of class (ii). For example, if the local distribution of sources domi- 
nates the spectrum beyond the suppression, as suggested by anisotropy studies, our new interpretation for the origin of the spectral cutoff explains naturally why the maximum energy observed on Earth coincides with that expected from a uniform distribution of sources but with UHECR nuclei propagating over cosmological distances. Moreover, the best fit to the observed spectrum and nuclear composition yields a proton maximum en$\operatorname{ergy} E_{\max }^{p}=E_{\max } / Z \sim 10^{9.5} \mathrm{GeV}$ [2]. This in turn gives a maximum energy for CNO species of $E_{\max }^{\mathrm{CNO}} \sim 10^{10.5} \mathrm{GeV}$, which is below the observed suppression in the energy spectrum, and therefore below the energy cutoff in the anisotropy analysis of [8]. Now, the typical values of the deflections of UHECRs crossing the Galaxy are $\theta \sim 10^{\circ} Z\left(10^{10} \mathrm{GeV} / E\right)$, and therefore it is challenging to accommodate anisotropy patterns with $Z \gtrsim 8$ nuclei [16]. As we have shown, $\mathrm{CNO}$ species can be accelerated in starburst superwinds to the maximum observed energies.

Altogether, this provides a compelling case demonstrating that there is strong evidence favoring UHECRs origin in starburst superwinds.

Work supported by NSF Grant PHY-1620661 and NASA Grant 80NSSC18K0464.

\section{References}

[1] L. A. Anchordoqui, G. E. Romero and J. A. Combi, Phys. Rev. D 60, 103001 (1999); L. A. Anchordoqui, H. Goldberg and D. F. Torres, Phys. Rev. D 67, 123006 (2003); L. A. Anchordoqui, Phys. Rev. D 97, no. 6, 063010 (2018).

[2] L. A. Anchordoqui, Phys. Rep. 801, 1 (2019).

[3] R. L. White, Astrophys. J. 289, 698 (1985); P. Schneider, Astron. Astrophys. 278, 315 (1993); M. H. Pope and D. B. Melrose, Publ. Astron. Soc. Austral. 11, 175 (1994).

[4] J. H. Matthews et al., Mon. Not. Roy. Astron. Soc. 479, no. 1, L76 (2018).

[5] K. Greisen, Phys. Rev. Lett. 16, 748 (1966); G. T. Zatsepin and V. A. Kuzmin, JETP Lett. 4, 78 (1966) [Pisma Zh. Eksp. Teor. Fiz. 4, 114 (1966)].

[6] D. Allard, N. G. Busca, G. Decerprit, A. V. Olinto and E. Parizot, JCAP 0810, 033 (2008); R. Aloisio, V. Berezinsky and A. Gazizov, Astropart. Phys. 34, 620 (2011).

[7] A. M. Taylor, M. Ahlers and D. Hooper, Phys. Rev. D 92, no. 6, 063011 (2015).

[8] A. Aab et al. [Pierre Auger Collaboration], Astrophys. J. 853, L29 (2018).

[9] R. U. Abbasi et al. [TA Collaboration], Astrophys. J. 867, L27 (2018).

[10] R. U. Abbasi et al. [TA Collaboration], Astrophys. J. 790, L21 (2014).

[11] A. M. Hillas, Ann. Rev. Astron. Astrophys. 22, 425 (1984).

[12] T. M. Heckman, L. Armus and G. K. Miley, Astrophys. J. Suppl. 74, 833 (1990).

[13] T. A. Thompson et al., Astrophys. J. 645, 186 (2006).

[14] J. D. Bregman, P. Temi, and D. Rank, Astron. Astrophys. 355, 525 (2000).

[15] L. A. Anchordoqui, D. Hooper, S. Sarkar and A. M. Taylor, Astropart. Phys. 29, 1 (2008); E. R. Owen, K. Wu, X. Jin, P. Surajbali and N. Kataoka, arXiv:1905.00338.

[16] R. C. dos Anjos et al., Phys. Rev. D 98, no. 12, 123018 (2018). 